\section{Keine Magensonde bei schwerer Demenz}

\author{
Ärzte sollten bei alten Patienten mit Schluckproblemen möglichst früh an \\ eine Magensonde denken, aber in der terminalen Phase keine neue PEG \\ legen. Vor allem bei fortgeschrittener Demenz macht die PEG keinen Sinn, \\ hieß es auf dem DGN-Kongress.
}

n Wilhelm Tell heißt es: „Rasch tritt der Tod den Menschen an, es ist ihm keine Frist gegeben, es stürzt ihn mitten in der Bahn, es reißt ihn fort vom vollem Leben“. Doch so unvermittelt wie zu Schillers Zeiten greift der Schnitter in der industrialisierten Welt nur noch selten zu. Ganz im Gegenteil. „Zwei Drittel aller Todesfälle sind heute absehbar“, erläuterte Professor Stefan Lorenzl, Paracelsus Universität Salzburg, mit Blick auf die Einsichten des großen deutschen Dichters. Ärzte in Kliniken müssen sich daher mit der Frage auseinandersetzen, wie sie sich verhalten sollen, wenn der Tod vor der Tür steht. In Umfragen gibt knapp die Hälfte der Klinikärzte zu, dass sie sich in solchen Fragen sehr unsicher fühlen - neurologische Chefärzte nicht ausgenommen: $60 \%$ gaben an, Angst vor den Rechtsfolgen beim Abbruch lebenserhaltender Maßnahmen zu haben. Dabei gehören Entscheidungen über eine Therapiebegrenzung für viele Ärzte zum Alltag, so Lorenzl (Abb. 1).

* Leitfaden des Bayerischen Landespflegeausschusses, Dezember 2008. http://www.pflegeservice-bayern.de/clients/mdk bayern/webcmspsb/CMS2Content.nsf/res/kuern-leitfaden. pdf/\$FILE/kuern-leitfaden.pdf

\section{PEG bringt nichts in der letzten Lebensphase}

Besonders schwer tun sich Ärzte mit der Begrenzung der Nahrungs- und Flüssigkeitszufuhr. Daher werden selbst bei terminal Kranken oft noch Magensonden gelegt - einen erheblichen Teil der jährlich rund 140.000 Sonden erhalten Demenzkranke. Lorenzl erinnerte jedoch daran, dass es in der letzten Lebensphase zu einer katabolen Stoffwechsellage kommt, an der auch eine hyperkalorische Ernährung durch eine perkutane endoskopische Gastrostomie (PEG) nichts ändere. Ein Gewichtsverlust sei in dieser Phase nicht zu verhindern, normale Nahrungsmengen könnten nicht mehr verarbeitet werden. In einer Untersuchung aus dem Jahr 2003 starben $85 \%$ der Patienten, die eine Magensonde zur Lebensverlängerung abgelehnt hatten, friedlich innerhalb von zwei Wochen. Eine weitere Studie in $2005 \mathrm{kam}$ zu dem Resultat: Demenzkranke, die zu erkennen gaben, dass sie keine Nahrung mehr wollten, und nicht künstlich ernährt wurden, waren zufriedener, hatten weniger Schmerzen und litten deutlich weniger, sagte Lorenzl. „Studien zeigen keine Hinweise auf eine Lebensverlänge-

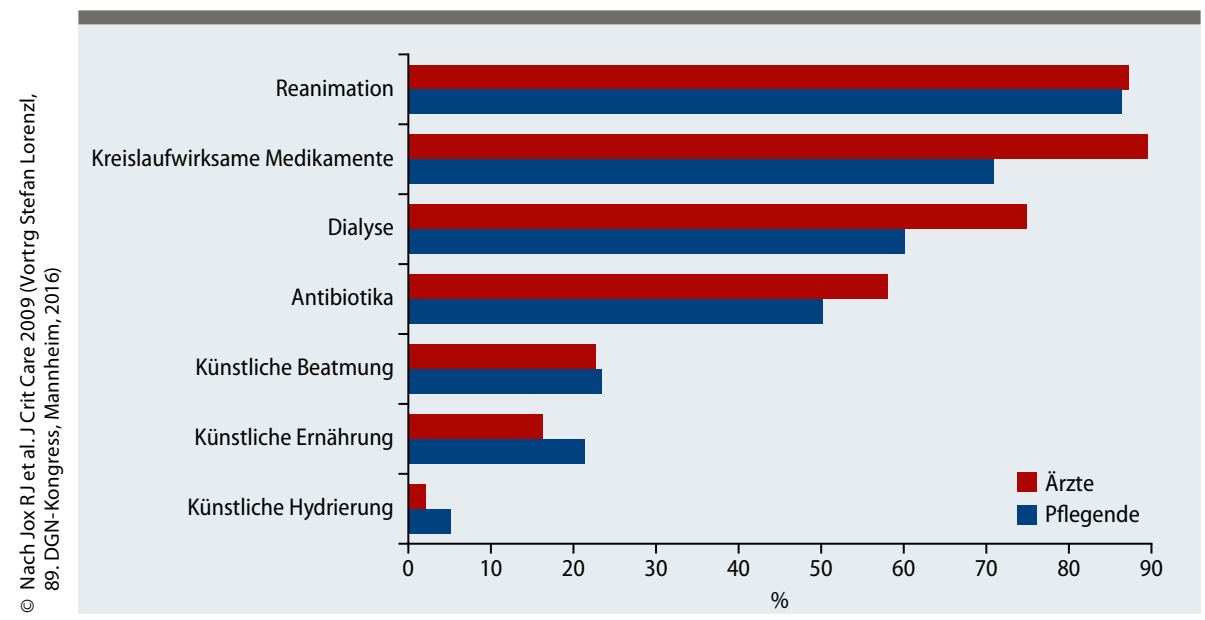

Abb. 1: Therapieformen, die begrenzt werden.

rung, eine Verbesserung des Ernährungsstatus, der Lebensqualität oder der Wundheilung. Dem stehen Nebenwirkungen der PEG wie Entzündungen, Verlust der Freude am Essen und eine Verringerung der pflegerischen Zuwendung gegenüber." Dennoch sei die künstliche Ernährung inzwischen fast Standard bei schwer Demenzkranken. Lorenzl verwies auf eine Untersuchung, nach der mehr als die Hälfte der schwer Demenzkranken nach Anlage einer PEG innerhalb eines Monats starben, und $90 \%$ innerhalb eines Jahres. Auch ein Cochrane-Review von 452 Studien fand weder einen Hinweis auf eine Lebensverlängerung noch auf eine Verbesserung des Ernährungsstatus durch die Magensonde bei fortgeschrittener Demenz. "Letztlich gibt es keine Studienevidenz, die hier die PEG-Anlage unterstützt“, meinte der Palliativmediziner. Er kritisierte daher die Leitlinie der „European Society for Clinical Nutrition and Metabolism“ (ESPEN) zur parenteralen Ernährung aus 2009. Sie hält die PEG für eine sichere und effektive Behandlung, die auch in der Terminalphase sterbender oder demenzkranker Patienten erwogen werden solle. „Hier haben wohl einige die Studien nicht gelesen.“

Für Lorenzl ist es wichtig, bei der PEG nach der medizinischen Indikation $\mathrm{zu}$ fragen. Haben etwa Patienten mit atypischem Parkinson-Syndrom deutliche Schluckstörungen oder Husten beim Essen, sei möglichst früh über eine Magensonde nachzudenken, „solange sie noch davon profitieren “. In der Sterbephase rät er, die Sonde dann zu belassen und nicht ohne Not zu entfernen.

Durch eine gute Pflege könne eine PEG oft vermieden werden, etwa indem Flüssigkeit angedickt und die Gabel nicht überladen werde. Auch Halsbeugemanöver helfen beim Schlucken. Allerdings würde dafür in Pflegeeinrichtungen oft nicht ausreichend Zeit aufgebracht, so Lorenzl. Eine gute Orientierung zur künstlichen Ernährung liefert seiner Auffassung nach ein Leitfaden des Bayerischen Landespflegeausschusses*.

Thomas Müller

„Palliativmedizin in der Neurologie. PEG ja oder nein?", DGN-Kongress 2016, Mannheim, 21. -24.9 .2016$ 